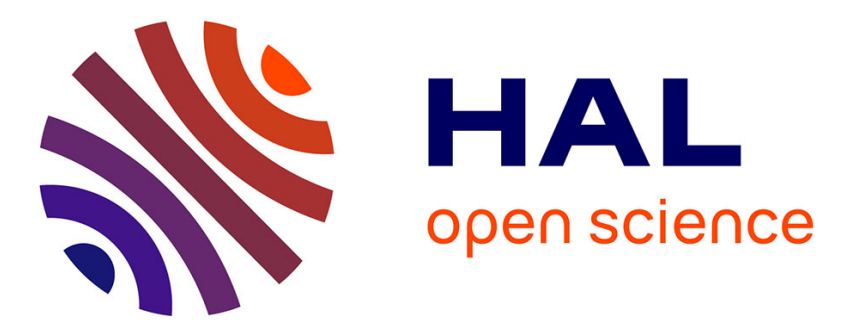

\title{
Optimized reducing-end labeling of cellulose nanocrystals: Implication for the structure of microfibril bundles in plant cell walls
}

Fangbo Lin, Jean-Luc Putaux, Bruno Jean

\section{- To cite this version:}

Fangbo Lin, Jean-Luc Putaux, Bruno Jean. Optimized reducing-end labeling of cellulose nanocrystals: Implication for the structure of microfibril bundles in plant cell walls. Carbohydrate Polymers, 2021, 257, pp.117618. 10.1016/j.carbpol.2021.117618. hal-03149209

\author{
HAL Id: hal-03149209 \\ https://hal.science/hal-03149209
}

Submitted on 22 Feb 2021

HAL is a multi-disciplinary open access archive for the deposit and dissemination of scientific research documents, whether they are published or not. The documents may come from teaching and research institutions in France or abroad, or from public or private research centers.
L'archive ouverte pluridisciplinaire HAL, est destinée au dépôt et à la diffusion de documents scientifiques de niveau recherche, publiés ou non, émanant des établissements d'enseignement et de recherche français ou étrangers, des laboratoires publics ou privés. 
1 Optimized reducing-end labeling of cellulose nanocrystals: implication for the structure of microfibril bundles in plant cell walls

\author{
Fangbo LIN, ${ }^{1}$ Jean-Luc PUTAUX ${ }^{1}$ and Bruno JEAN ${ }^{1 *}$
}

${ }^{1}$ Univ. Grenoble Alpes, CNRS, CERMAV, F-38000 Grenoble, France

\begin{abstract}
A strategy to optimize the labeling of the reducing end of native cellulose nanocrystals (CNCS) with gold nanoparticles (AuNPs) was developed and used to investigate the arrangement of the elementary crystallites constituting these biosourced particles. First, CNCs pre-functionalized with thiosemicarbazide molecules were reacted with presynthesized AuNPs. A second method consisted in synthesizing AuNPs in situ from soluble gold derivatives in the presence of CNCs regioselectively functionalized with thiosemicarbazide molecules. Transmission electron microscopy images revealed that the direct reaction resulted in a low labeling yield and the undesired formation of AuNP aggregates. Oppositely, unprecedent high labeling yields were achieved through the in situ growth approach, with a vast majority of CNCs bearing one or several AuNPs on one end. These results evidence that cotton-derived CNCs are composed of the unidirectional assembly of chemically polar elementary crystallites, implying that the acid hydrolysis isolates fragments of microfibril bundles present in the cell walls.
\end{abstract}

\title{
1. Introduction
}

Cellulose nanocrystals (CNCS) consist of biobased nanorods produced by acid hydrolysis of cellulose substrates, such as microcrystalline cellulose, cotton linters, pulp or paper (Habibi, Lucia, \& Rojas, 2010; Klemm et al., 2018). These slender nanoparticles, with a size depending on the source of cellulose and hydrolysis conditions (Brito, Pereira, Putaux, \& Jean, 2012; Dufresne, 2012; ElazzouziHafraoui et al., 2008), are renewable and biocompatible. They display tunable aspect ratios, a low density, a high specific surface area (Angles \& Dufresne, 2001; Ruiz, Cavaille, Dufresne, Gerard, \& Graillat, 2000), a remarkable high tensile strength and elastic modulus (Eichhorn et al., 2010; Eichhorn, Young, \& Davies, 2005; Sturcova, Davies, \& Eichhorn, 2005), and can self-organize into liquid crystalline phases (Lagerwall et al., 2014; Revol, Bradford, Giasson, Marchessault, \& Gray, 1992). As a result, CNCs can be exploited in various fields such as nanocomposites, packaging, paints, oil recovery, etc. (Habibi 
et al., 2010; Klemm et al., 2018; Yang, Shi, Zhitomirsky, \& Cranston, 2015). As of today, these nontoxic biosourced particles are no longer only produced at the laboratory scale, but pilot-plant quantities are commercially available (Bras \& Chauve, 2014).

The two ends of a cellulose chain are not identical since the so-called non-reducing end exhibits a secondary hydroxyl group, while the reducing end displays a hemiacetal cyclic moiety, in equilibrium with a highly reactive aldehyde form. In CNCs prepared from native cellulose, the chains are crystallized in a parallel arrangement that corresponds to the so-called "cellulose I" allomorph (Koyama, Helbert, Imai, Sugiyama, \& Henrissat, 1997; Nishiyama, Langan, \& Chanzy, 2002). In these CNCs, the chemical polarity of each chain is transmitted to the nanocrystal which, accordingly, has a non-reducing end rich in secondary hydroxyl groups and a reducing end covered with aldehyde moieties. In other terms, CNCs are chemically polar rodlike particles. This property was evidenced by specific staining of the reducing ends or the enzymatic degradation of the non-reducing counterparts (Chanzy \& Henrissat, 1985; Hieta, Kuga, \& Usuda, 1984). This unique feature of CNCs has motivated a growing, yet still limited, number of research works aiming at regioselectively modifying the reducing end of CNCs, which were recently reported in two reviews (Heise et al., 2020; Tao, Lavoine, Jiang, Tang, \& Lin, 2020). Such an asymmetric derivatization of CNCs is highly appealing since it can lead to a variety of innovative behaviors that pave the way to the use of these Janus-like particles in advanced applications. However, this localized chemical modification targeting a very small region of the whole object inherently suffers from low reactivity and steric hindrance issues and thus raises serious concerns about a quantitative physical and chemical characterization of the derivatized samples.

The combination of CNCs with metal nanoparticles (NPs) is a way to obtain hybrid organicinorganic materials benefiting from the properties of both components and expectedly prone to offer new synergistic behaviors. Works have thus been dedicated to the association of both types of particles. For example, thanks to their large specific surface area and their colloidal stability in water, CNCs have been used as efficient stabilizers for many metal NPs, such as Pd (Wu et al., 2013), Ag (Shi et al., 2015; Tang, Shi, Berry, \& Tam, 2015) and Au (Chen, Cao, Quinlan, Berry, \& Tam, 2015; Wu et al., 2014; Yokota, Kitaoka, Opietnik, Rosenau, \& Wariishi, 2008; Zhang, Sebe, Wang, \& Tam, 2018). Semenikhin et al. (Semenikhin, Kadasala, Moon, Perry, \& Sandhage, 2018) have prepared individually dispersed gold nanoshell-bearing CNCs by using gold nanoparticles. Different attempts specifically targeting the reducing end of CNCs have also been reported. Hieta et al. (Hieta et al., 1984) stained the reducing ends of Valonia CNCs with silver nanoparticles using a reductive amination reaction. Kuga et al. (Kuga \& Brown, 1988) and Koyama et al. (Koyama, Sugiyama, \& Itoh, 1997) have used a silver-staining technique as well to identify the reducing end of CNCs from Valonia, ramie and bacterial cellulose. The reducing end of CNCs prepared from Valonia and ramie has also been stained with gold NPs to 
demonstrate the molecular directionality (Kim, Imai, Wada, \& Sugiyama, 2006). Lokanathan et al. (Lokanathan, Lundahl, Rojas, \& Laine, 2014; Lokanathan et al., 2013) have produced cilia-mimetic hairy surfaces, based on end-immobilized nanocellulose at gold surfaces by an oxidation followed by a peptide coupling reaction. More recently, Li et al. (L. Li et al., 2018) have used a triazole end-grafting reaction on CNCs for water-redispersion improvement using gold NPs. Nevertheless, judging from the reported transmission electron microscopy (TEM) images shown in the aforementioned works and based on reported low labeling yield values of only $15 \%$, efficient methods to regioselectively label the reducing end of CNCs still need to be developed.

In the present paper, we propose to tackle the optimization of the regioselective labeling of CNCS with gold nanoparticles (AuNPs), which stands as a prerequisite to fully benefit from synergistic properties of materials comprising such hybrids. The work is based on the use of an alternative labeling strategy avoiding the use of pre-synthesized AuNPs and associated steric hindrance issues, and thus prone to favor high labeling yields. Two types of native CNCs from different cellulose sources were used. Complementary TEM, dynamic light scattering, small-angle X-ray scattering and UV-Vis spectroscopy analyses were used to characterize the labeled particles. Such a strategy additionally allowed us to give some new insight into the basic characteristics of these particles and their parent microfibrils.

\section{Experimental section}

\subsection{Materials}

Cotton linters were provided by Buckeye Cellulose Corporation and used as the cellulose source without any further purification. Acetic acid (HAc) was purchased from Carlo Erba Reagents and other chemicals were purchased from Sigma-Aldrich and used as received. Deionized water was used in all experiments.

\subsection{Preparation of CNCs}

Native CNCs from cotton (cCNCS). Cotton linters were treated with 65 wt. \% sulfuric acid during $30 \mathrm{~min}$ at $63^{\circ} \mathrm{C}$ under mechanical stirring following the protocol described by Revol et al. (Revol et al., 1992). The resulting suspension was washed by repeated centrifugation/redispersion cycles and dialyzed against distilled water until the conductivity of the dialysis bath reached the conductivity of distilled water. In the following step, the suspension was ultrasonicated for 4 min with a Branson 250 digital sonifier and successively filtered through $8 \mu \mathrm{m}$ and $1 \mu \mathrm{m}$ cellulose nitrate membranes using a Sartorius filtration equipment. These native cellulose CNCs obtained from cotton will be referred to as cCNCs. 
Native CNCs from wood (wCNCS). A 3 wt. \% aqueous dispersion of neutral CNCs was provided by Melodea Ltd. (Israel) and used after dilution to $1 \mathrm{wt}$. \% with deionized water. These nanocrystals, that will be referred to as wCNCs, were produced from wood by the reported sulfuric acid (64\%) hydrolysis method (Bondeson, Mathew, \& Oksman, 2006).

\subsection{Sulfur content}

A CNC suspension containing about $100 \mathrm{mg}$ of cellulose was titrated with a solution of $0.01 \mathrm{M}$ $\mathrm{NaOH}$ using a MeterLab CDM 210 conductivity meter. At the equivalence, we have:

$$
V_{e q} \times C_{N a O H}=n_{\text {cellulose }} \times d_{S}
$$

with $V_{e q}$ the added volume of $\mathrm{NaOH}, n_{\text {cellulose }}$ the number of moles of anhydroglucose units (AGU) and $d_{S}$ the degree of substitution representing the number of sulfate groups per AGU. We know that

$$
n_{\text {cellulose }}=\frac{m_{\text {cellulose }}}{M_{\text {ave }}}
$$

where $m_{\text {cellulose }}$ is the dry weight of introduced CNC and $M_{\text {ave }}$ is the average molar mass of a substituted glucose residue which is given by the following relation:

$$
M_{\text {ave }}=162 \times\left(1-d_{S}\right)+242 d_{S} \quad \text { (Equation 3) }
$$

where $162 \mathrm{~g} \mathrm{~mol}^{-1}$ et $242 \mathrm{~g} \mathrm{~mol}^{-1}$ are respectively the molar masses of one AGU and a mono-sulfated unit. Equations 1 and 2 allow us to write:

$$
d_{S}=\frac{V_{e q} \times C_{N a O H} \times M_{\text {ave }}}{m_{\text {cellulose }}}
$$

After solving Equations 3 and 4, $M_{\text {ave }}$ was calculated from the following relation:

$$
M_{\text {ave }}=\frac{162}{1-80 \times \frac{V_{e q} \times C_{N a O H}}{m_{\text {cellulose }}}}
$$

The sulfur content, $\tau_{S}$, was then calculated using the following relation:

$$
\tau_{S}=\frac{32 \times d_{S}}{M_{\text {ave }}}
$$

(Equation 6)

\subsection{Aldehyde content}

The Fehling test was used to measure the aldehyde content (Vollhardt \& Schore, 2014). Two A and B solutions were prepared. For solution $\mathrm{A}, 69.28 \mathrm{~g}$ of $\mathrm{CuSO}_{4} \bullet 5 \mathrm{H}_{2} \mathrm{O}$ was dissolved in $1 \mathrm{~L}$ of distilled water. Solution B was prepared by dissolving $346 \mathrm{~g}$ of $\mathrm{KNaC}_{4} \mathrm{H}_{4} \mathrm{O}_{6} \bullet 4 \mathrm{H}_{2} \mathrm{O}$ and $120 \mathrm{~g}$ of $\mathrm{NaOH}$ in $1 \mathrm{~L}$ of distilled water. The final solution for the titration was obtained by mixing these two solutions in equal volume. Two grams of freeze-dried CNCs were dispersed into $50 \mathrm{~mL}$ of deionized water. The suspension was heated to $65^{\circ} \mathrm{C}$ with a very gentle stirring and then titrated dropwise with the titration solution using a micropipette until there was no more red precipitate. After each drop, about 2 min were allowed for 
the detection of the red precipitate.

The net reaction between an aldehyde and the copper (II) ions in Fehling's solution can be written as:

$$
\mathrm{RCHO}+2 \mathrm{Cu}\left(\mathrm{C}_{4} \mathrm{H}_{4} \mathrm{O}_{6}\right)_{2}^{2-}+5 \mathrm{OH}^{-} \rightarrow \mathrm{RCOO}^{-}+\mathrm{Cu}_{2} \mathrm{O}+\mathrm{C}_{4} \mathrm{H}_{4} \mathrm{O}_{6}^{2-}+3 \mathrm{H}_{2} \mathrm{O}
$$

At the equivalence, we have:

$V_{e q} \times c_{C u\left(C_{4} H_{4} O_{6}\right)_{2}^{2-}}=n_{C u\left(C_{4} H_{4} O_{6}\right)_{2}^{2-}}=2 n_{R C H O} \quad$ (Equation 7)

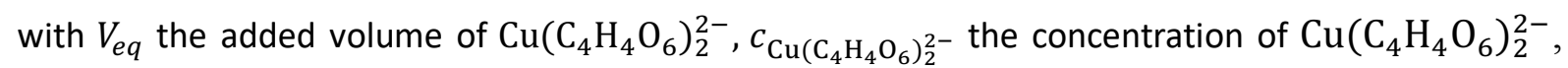
$n_{\mathrm{Cu}\left(\mathrm{C}_{4} \mathrm{H}_{4} \mathrm{O}_{6}\right)_{2}^{2-}}$ the number of moles of added $\mathrm{Cu}\left(\mathrm{C}_{4} \mathrm{H}_{4} \mathrm{O}_{6}\right)_{2}^{2-}$ and the $n_{R C H O}$ the number of moles of aldehyde groups that we want to deduce.

\subsection{Thioureation}

Thioureation refers to the reaction that introduces the thiourea group. An aqueous solution containing $10 \mathrm{wt}$ \% HAc and $2 \mathrm{wt}$. \% thiosemicarbazide was prepared. Then, a suspension containing about $2 \mathrm{~g}$ of cellulose was mixed with the previous solution in the same volume in a flask surmounted by a condenser. The mixture was heated at $60^{\circ} \mathrm{C}$ for $3 \mathrm{~h}$ and finally cooled in ice. Then, $\mathrm{NaCl}$ was added until its concentration was approximately $0.4 \mathrm{M}$. The suspension was then centrifuged for $30 \mathrm{~min}$ at $11200 \mathrm{rpm}$ and the modified CNCs were redispersed in HAc. The latter operation was repeated twice. The final product, hereafter referred to as S-e-CNC, was dialyzed against distilled water until constant conductivity of the dialysis bath and ultrasonicated for 4 min with a Branson 250 digital sonifier.

\subsection{Preparation of gold nanoparticles (AuNPs)}

Deionized water $(925 \mathrm{~mL})$ was heated to $95^{\circ} \mathrm{C}$ and $92.5 \mathrm{mg}$ of chloroauric acid was added under stirring. Once $25 \mathrm{~mL}$ of a $1 \mathrm{wt}$. \% trisodium citrate solution was added, the mixture was stirred for 30 $\min$ at $95^{\circ} \mathrm{C}$ and then cooled down (Haiss, Thanh, Aveyard, \& Fernig, 2007).

\subsection{CNC labeling with gold nanoparticles}

Two methods were used to label CNCs with gold nanoparticles.

Direct reaction method: One liter of AuNP suspension containing $54 \mathrm{mg}$ of pure gold was mixed with $2 \mathrm{~g}$ of a 1 wt.\% S-e-CNC suspension. The mixture was stirred for 3 days away from light.

In situ growth method: Two grams of a 1 wt. \% S-e-CNC suspension was added into $925 \mathrm{~mL}$ deionized water. The suspension was then heated to $95^{\circ} \mathrm{C}$. $92.5 \mathrm{mg}$ of chloroauric acid and $25 \mathrm{~mL}$ of a $1 \mathrm{wt} . \%$ trisodium citrate aqueous solution were then added under stirring for $30 \mathrm{~min}$ at $95{ }^{\circ} \mathrm{C}$. The resulting suspension was then cooled down to room temperature.

\subsection{Dynamic light scattering (DLS)}

DLS experiments were carried out with a Malvern NanoZS instrument. All measurements were 
made at a well-controlled $\left( \pm 0.05^{\circ} \mathrm{C}\right)$ temperature with a $632.8 \mathrm{~nm}$ laser at a backscattering detection angle of $173^{\circ}$. The intensity size distribution was obtained from the analysis of the correlation function using the multiple narrow mode algorithm of the Malvern DTS software.

\subsection{UV-Vis absorption spectroscopy}

The measurements were performed on a CARY 50 spectrophotometer using 0.5 wt. \% samples and the wavelength scan mode (from 400 to $800 \mathrm{~nm}$ ) at $25^{\circ} \mathrm{C}$.

\subsection{Transmission electron microscopy (TEM)}

Drops of about 0.001 wt. \% CNC aqueous suspensions were deposited onto carbon-coated TEM grids freshly glow-discharged in an easiGlow plasma cleaning system (Pelco). After 2 min, the liquid in excess was blotted away with a filter paper and, prior to drying, a drop of a 2 wt. \% uranyl acetate aqueous solution was deposited onto the specimen. After 2 min, the stain in excess was wicked off, and the remaining thin liquid film allowed to dry. The specimens were observed either with a Philips CM200 'Cryo' or a JEOL JEM 2100-Plus microscope operated at $200 \mathrm{kV}$. The images were recorded with TVIPS F216 TemCam and Gatan Rio 16 digital cameras, respectively. Size distribution plots were obtained using the ImageJ software. After labeling with AuNPs, a statistical analysis of the labeling yield was performed using the ImageJ software based on the observation of a statistically relevant number of objects (between 200 and 600).

\subsection{Zeta potential}

The zeta potential of CNCs was measured from 0.1 wt. \% suspensions by electrophoresis coupled with laser Doppler velocimetry using a Malvern NanozS instrument. Samples contained $10 \mathrm{mM} \mathrm{NaCl}$. Data were averaged over 3 measurements, each measurement comprising 10 subruns.

\subsection{Small-angle X-ray scattering (SAXS)}

Samples were measured using a Xeuss 3.0 apparatus (SAXS Lab, Saclay, France) equipped with a micro-focused Cu K $\alpha$ source (wavelength of $1.54 \AA$, 8 keV) and a Pilatus detector (Dectris, Switzerland). The distance between the sample and detector was $2.48 \mathrm{~m}$, which corresponds to a Q-range from 0.0045 to $0.25 \AA^{-1}$. An X-ray flux of about $7 \times 10^{6}$ photons per second was provided by a collimated beam size of $0.5 \times 0.6 \mathrm{~mm}^{2} .1 .5 \mathrm{~mm}$ external diameter glass capillaries (0.01 mm wall thickness) were used as sample containers. Independent measurements of solvent, empty capillary and dark field were carried out for data subtraction purposes. The incoming data were normalized to absolute units.

The scattering intensity as a function of Q generally follows.

$$
I(Q)=c \cdot \Delta \rho^{2} \cdot P(Q) \cdot S(Q)
$$

(Equation 8)

where I (q) the scattering intensity, c the sample concentration, $P(Q)$ the form factor, $S(Q)$ the structure factor, $\Delta \rho^{2}$ the contrast between sample and solvent, $Q=\frac{4 \pi \sin \theta}{\lambda}$ the norm of the scattering vector 
with $\vartheta$ the scattering angle and $\lambda$ the wavelength of incoming X-ray.

The contrast term is defined as the square of the scattering length density difference between the sample and the solvent, i.e.

$$
\Delta \rho^{2}=\left(\rho_{\text {sample }}-\rho_{\text {solvent }}\right)^{2}
$$

Scattering length densities of cellulose, gold nanoparticles and water are equal to $14.5 \times 10^{-6} \AA^{-2}$ and $12.5 \times 10^{-5} \AA^{-2}$, and $9.47 \times 10^{-6} \AA^{-2}$, respectively, leading to

$\Delta \rho_{\text {AuNP/solvent }}^{2}=1.33 \times 10^{-8} \AA^{-4} \gg \Delta \rho_{C N C / \text { solvent }}^{2}=2.5 \times 10^{-11} \AA^{-4}$

(Equation 10)

Consequently, the SAXS intensity arising from an aqueous suspension containing comparable concentrations of CNCs and AuNPs will mostly be attributed to gold nanoparticles.

SAXS data were fitted by assuming that $S(Q)=1$ for the dilute suspensions probed and using the form factor of spheres given by

$$
P(Q)=\left[\frac{\sin (Q R)-Q R \cos (Q R)}{Q R^{3}}\right]^{2} \quad \text { (Equation 11) }
$$

The fit was achieved using the open source Sasview software (http://www.sasview.org/).

\section{Results}

\subsection{Morphology of the starting particles}

cCNCs resulting from the sulfuric acid hydrolysis of cotton linters consisted of rodlike nanoparticles with a number average length of $129 \mathrm{~nm}$ (standard deviation 47\%) and a number average width of 27 $\mathrm{nm}$ (standard deviation 46\%), as shown in Supplementary Material Figure S1. These CNCs are composed of a fascicle of laterally associated elementary crystallites (Figure 1a), in good agreement with literature reports (Elazzouzi-Hafraoui et al., 2008; Lokanathan et al., 2013). The average sulfur content obtained by conductometric titration was about $0.22 \mathrm{mmol} \mathrm{g}^{-1}$ and a zeta potential of $-34 \pm 5$ $\mathrm{mV}$ was measured, confirming that $\mathrm{CCNCs}$ possess negative sulfate half-esters on their surface.

The TEM micrograph of wCNCs shown in Figure $1 \mathrm{~b}$ reveals that these nanoparticles are morphologically different from cCNCs. wCNCs are indeed rodlike but are longer (number average length of $227 \mathrm{~nm}$ and standard deviation of 23\%) and thinner (number average width of $14 \mathrm{~nm}$ and standard deviation of 32\%) than cCNCs (size distribution plots shown in Supplementary Material Figure S2). Their average aspect ratio is about 16, compared to about 5 for cCNCs. In contrast with cCNCs, wCNCs comprise only one or two elementary crystallites. A sulfur content of $0.325 \mathrm{mmol} \mathrm{g}^{-1}$ has been reported by the manufacturer (Rivkin et al., 2015) and a zeta-potential of $-33.7 \pm 5 \mathrm{mV}$ was measured using the previously described method. The negative surface charges on cCNCs and wCNCs generate 

absence of aggregates.
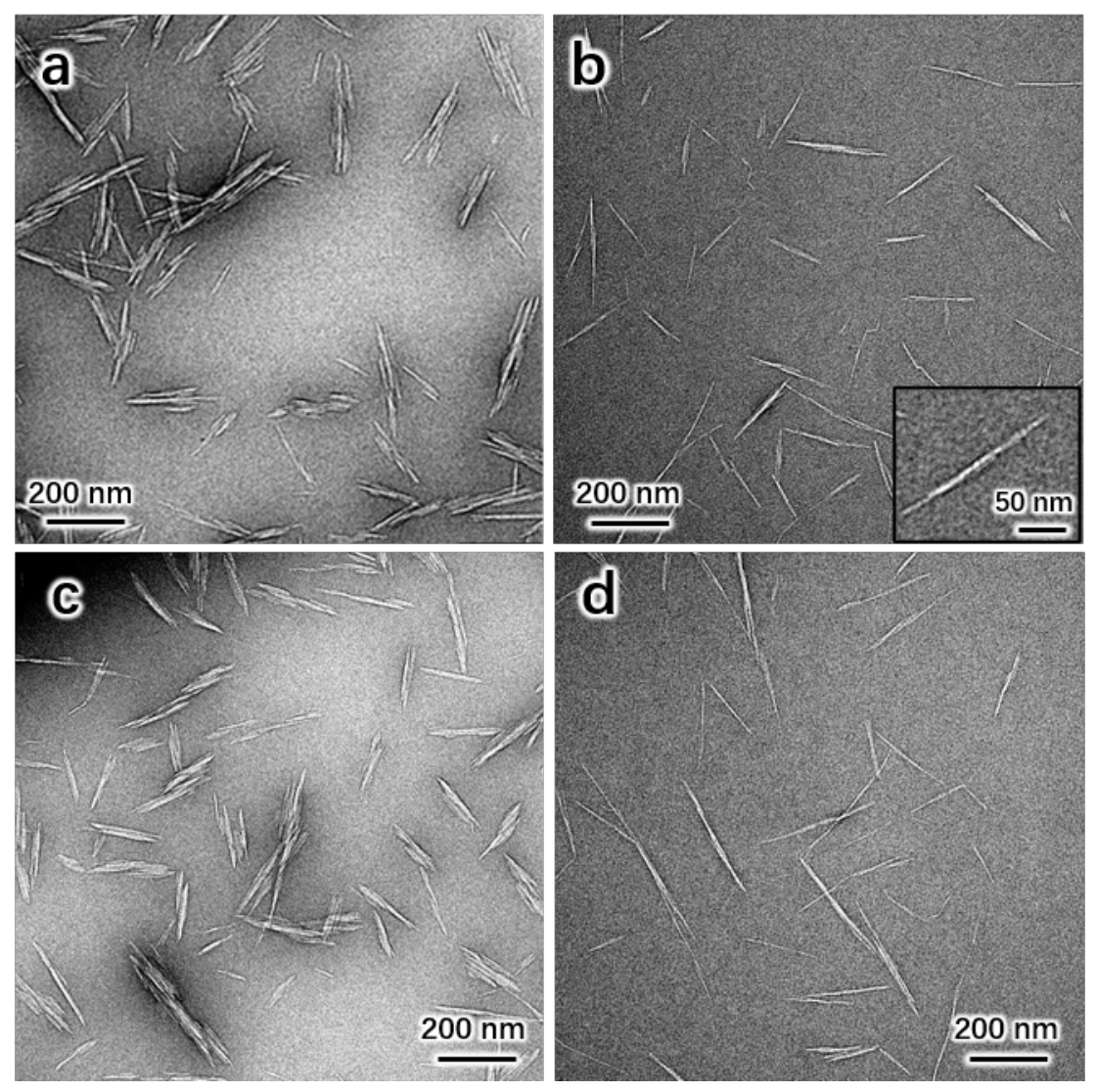

Figure 1. TEM micrographs of negatively stained cCNCs (a), wCNCs (b), S-e-cCNCs (c), S-e-wCNCs (d) prepared from aqueous suspensions at room temperature.

An aqueous colloidal suspension of AuNPs was prepared from the sodium citrate-stabilization method (Haiss et al., 2007). One minute after addition of $\mathrm{Na}_{3} \mathrm{C}_{6} \mathrm{H}_{5} \mathrm{O}_{7}$, the transparent solution turned into wine-red upon formation of gold colloids. The UV-Vis spectrum showed an absorption peak at about $525 \mathrm{~nm}$, indicative of a nanoparticle diameter between 10 and $30 \mathrm{~nm}$ (Supplementary Material Figure S3) (Haiss et al., 2007). A hydrodynamic diameter of $20 \pm 5 \mathrm{~nm}$ was determined by DLS (Supplementary Material Figure S4), in good agreement with the UV-Vis spectroscopy results.

\subsection{Nanocrystal prefunctionalization with sulfur atoms}

In order to promote the binding of AuNPs to CNCs, sulfur atoms were introduced by a one-step thioureation of the cellulose chain reducing ends, as described in Scheme 1. Samples prefunctionalized with $\mathrm{S}$ atoms are designated with the prefix "S-e-" (standing for sulfur at the reducing end). In the presence of acetic acid, the aldehyde groups react with thiosemicarbazide and produce an imine group. As the reducing ends represent only a few percent of the total CNC surface, techniques 
such as FTIR could not detect such small changes of functional groups due to a limited sensitivity. However, the initial and post-thioureation aldehyde contents of the two different $\mathrm{CNCs}$, i.e. the concentration of reducing ends before and after prefunctionalization were measured using the Fehling test and compared. The initial values were $24.9 \pm 0.4$ and $18.8 \pm 0.6 \mu \mathrm{mol} \mathrm{g}^{-1}$ for cCNCs and wCNCs, respectively. After the thioureation step, the aldehyde content decreased to $9.1 \pm 0.5$ and $6.9 \pm 0.6$ $\mu \mathrm{mol} \mathrm{g}{ }^{-1}$ for S-e-cCNCs and S-e-wCNCs, respectively. This decrease in the number of aldehyde groups shows that the thioureation reaction was successful. In both cases, the molar reaction yield was high and of about $64 \%$.
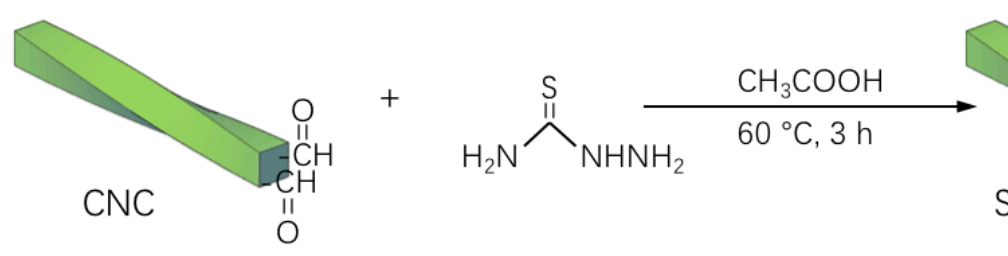

Scheme 1. Asymmetric thioureation of the reducing ends of CNCs during which the aldehyde groups are converted into imine groups.

Figures $1 \mathrm{c}$ and $1 \mathrm{~d}$ show TEM micrographs of the pre-functionalized CNCs. These nanocrystals are very similar to their parent particles, which reveals that the thioureation reaction did not alter their morphology.

\subsection{Regioselective labeling of CNCs with gold nanoparticles}

Using the thioureation reaction, sulfur atoms were successfully grafted on the reducing ends of CNCs, as indicated by the very significant decrease in aldehyde content. Starting from this prefunctionalized nanocrystals, the labeling with AuNPs relied on the well-known spontaneous reaction between sulfur and gold, which occurs through a $\sigma-\pi$ coordinate bond (Ning, Xie, Xing, Deng, \& Yang, 1996; Ulman, 1996). Two strategies, namely a direct reaction of S-e-CNCs with presynthesized AuNPs and an in situ growth method, consisting in synthesizing the gold colloids from soluble derivatives in the presence of S-e-CNCs were applied to functionalize the cCNCs with AuNPs. The two procedures, described in the Experimental Section, are depicted in Scheme 2. 


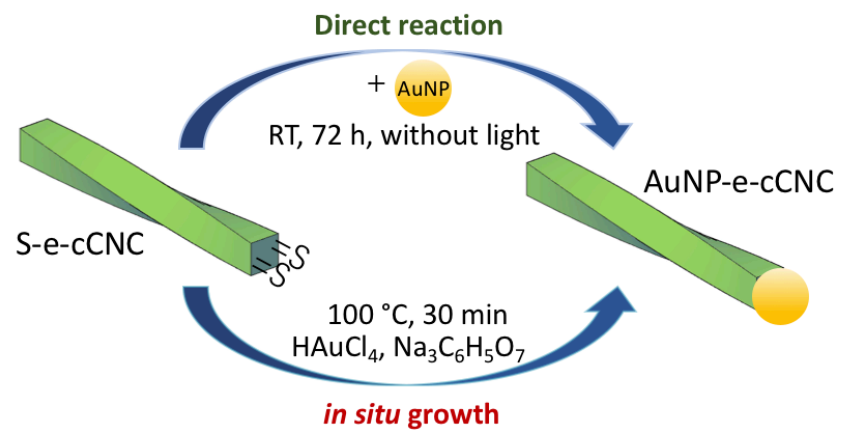

271

Scheme 2. Asymmetric functionalization of CNCs with gold nanoparticles with the direct reaction or in situ growth methods.

cCNCs that were end-functionalized with gold particles, hereafter referred to as AuNP-e-cCNCs, prepared by direct reaction and in situ growth, were first investigated by DLS. As shown in Figure 2a, the hydrodynamic diameter $\left(D_{h}\right)$ of presynthesized AuNPs was about $20 \mathrm{~nm}$ and that of S-e-cCNCs was about $91 \mathrm{~nm}$, which corresponds to the value usually reported for bare cotton CNCs prepared by sulfuric acid hydrolysis (Elazzouzi-Hafraoui et al., 2008; Lin, Cousin, Putaux, \& Jean, 2019). This $D_{h}$ indicated that the thioureation reaction had no effect on the size of CNCs, which was confirmed by the TEM images in Figure 1. For AuNP-e- cCNCs obtained by the direct reaction method, the intensity size distribution plot shows three peaks. The first one at $20 \mathrm{~nm}$ can be ascribed to the presence of individual AuNPs (not bonded to CNCs). The second one at $98 \mathrm{~nm}$ is slightly shifted to larger values when compared to the $D_{h}$ corresponding to as-prepared or sulfur-functionalized cCNCs (91 nm), which might indicate that a fraction of CNCs were labeled with AuNPs. Although it is difficult to fully trust the value corresponding to the third population at about $5000 \mathrm{~nm}$, which is at the upper confidence limit of the technique, this third signal indicates the presence of large-size aggregates.

Figure $2 \mathrm{~b}$ shows the intensity size distribution of a $0.1 \mathrm{wt}$. \% AuNP-e-cCNCs aqueous suspension resulting from the in situ growth strategy. When compared to the direct reaction case, several differences can be observed. First, a peak at about $17 \mathrm{~nm}$ is also observed, but its intensity is considerably lower. This signal can tentatively be attributed to the presence of a limited number of individual AuNPs, which would have a slightly smaller hydrodynamic diameter than the presynthesized ones. A second population with a $D_{h}$ of about $116 \mathrm{~nm}$ is detected. In contrast with the sample generated by direct reaction, this signal is clearly shifted when compared to the as-prepared cCNCs peak at $91 \mathrm{~nm}$, revealing that most particles have increased in size but without reaching a value large enough to account for aggregation. Such features are compatible with the sample being constituted of CNCs bearing AuNPs. No further peak is detected in the large size region, showing the absence of aggregates. 

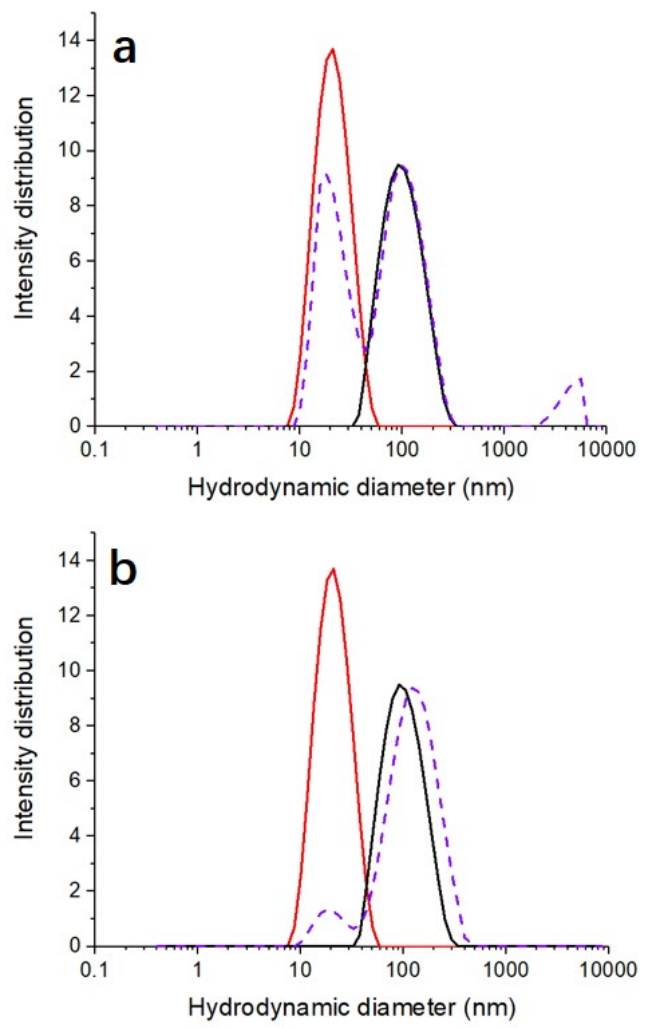

Figure 2. Intensity size distribution of a $0.5 \mathrm{wt}$.\% gold nanoparticles suspension (red solid line), $0.1 \mathrm{wt} . \%$ S-e-cCNCs suspension (black solid line) and 0.1 wt.\% AuNP-e-cCNCs suspension (violet dashed line) prepared by direct reaction (a) and in situ growth (b) measured by DLS at $20^{\circ} \mathrm{C}$.

In order to complement the results obtained by DLS, TEM was used to observe the samples prepared by the two different methods. In the TEM images shown in Figure 3, the AuNPs appear as dark spheres (due to the high electron density of gold) with an average diameter of about $20 \mathrm{~nm}$ and a narrow size distribution, irrespective of the method used to synthesize them. Figures $3 a$ and $3 b$ show the TEM micrographs of AuNP-e-cCNCs prepared by direct reaction. These images evidence that the sample comprises non-derivatized CNCs, free AuNPs gathered in clusters and a fraction of CNCs labeled with a AuNP. In the latter case, it is clear from Figure $3 \mathrm{~b}$ that the AuNP was attached at only one end of the crystals. Furthermore, in most cases, the AuNP attached at the end of the CNC is located at a specific position in direct alignment with the long axis of symmetry (c-axis) of one of the elementary crystallites constituting it. Rather similar results of relatively low efficiency of the labeling were already reported in the literature using different strategies. Indeed, in these works, observation of TEM images and some estimation indicated an about $15 \%$ labeling yield on the reducing end of CNCs, which is also the value that we achieved in the present study using the direct reaction method. 

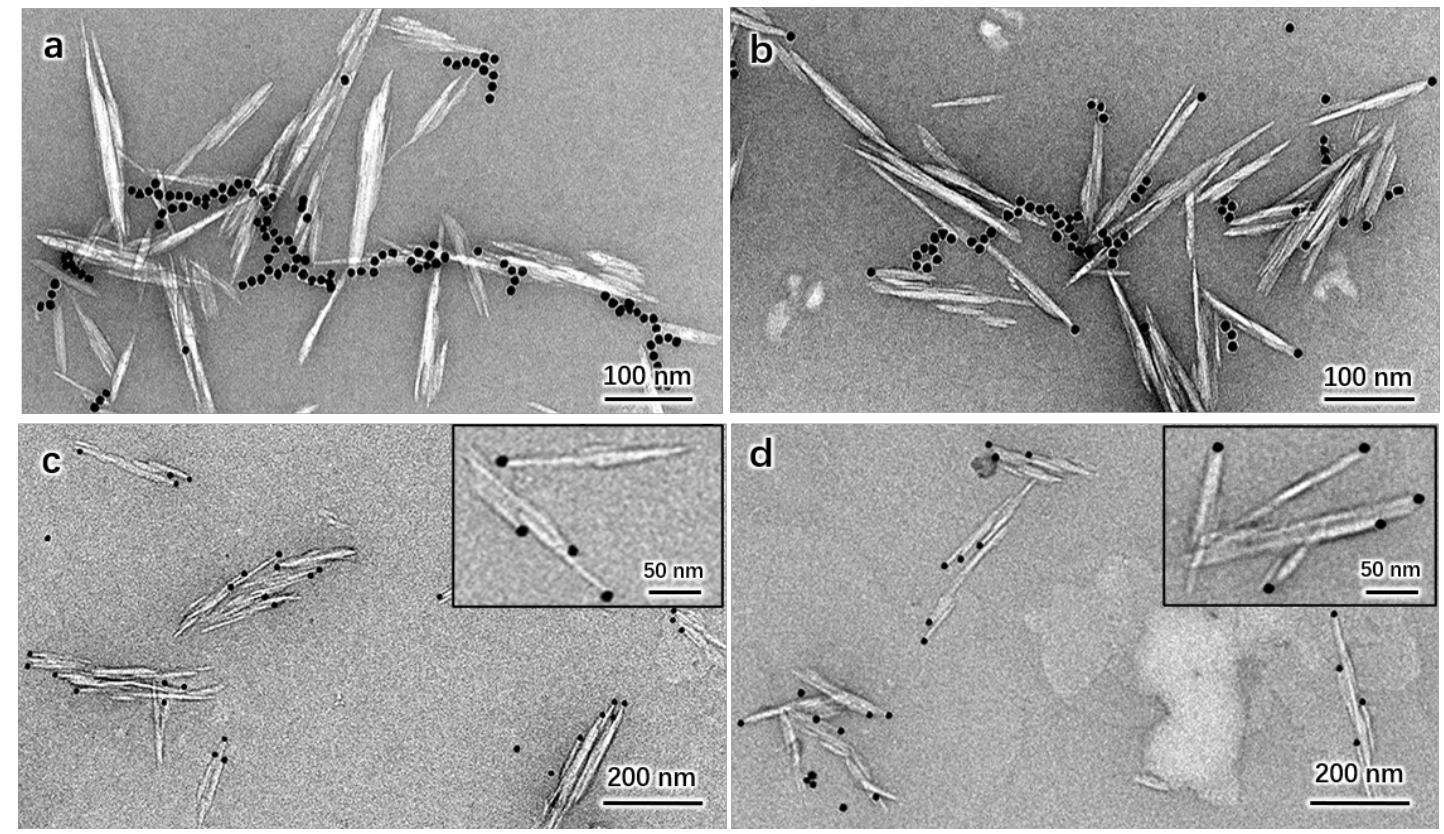

Figure 3. TEM micrographs of negatively stained AuNP-e-cCNCs prepared by direct reaction $(a, b)$ and in situ growth (c, d) from aqueous suspensions at room temperature.

In contrast, as shown in Figures $3 \mathrm{c}$ and $3 \mathrm{~d}$, a large number of cCNCs were functionalized with AuNPs when using the in situ growth strategy. It can indeed clearly be seen that most cCNCs are labeled with several AuNPs located on one end of the elementary crystallites constituting the CCNCs. Each elementary crystallite bears no more than one AuNP coaligned along the $c$-axis. Furthermore, for all hybrid particles, the AuNPs are systematically located on one side of the CNC. These results evidence a remarkable asymmetric functionalization. Moreover, when compared to the direct reaction method, a drastically reduced number of non-functionalized cCNCs and individual isolated AuNPs can be observed. Based on the observation of 32 TEM micrographs containing about 650 particles, a statistical analysis was performed to quantitatively characterize the grafting yield (Figure 4) that reached $81 \%$. This yield, 5 to 10 times higher than those previously obtained, shows the very high efficiency of the in situ growth method, which can be attributed to the preferential nucleation and growth of AuNPs at the sulfur-derivatized end of cCNCs acting as nucleating agents. 


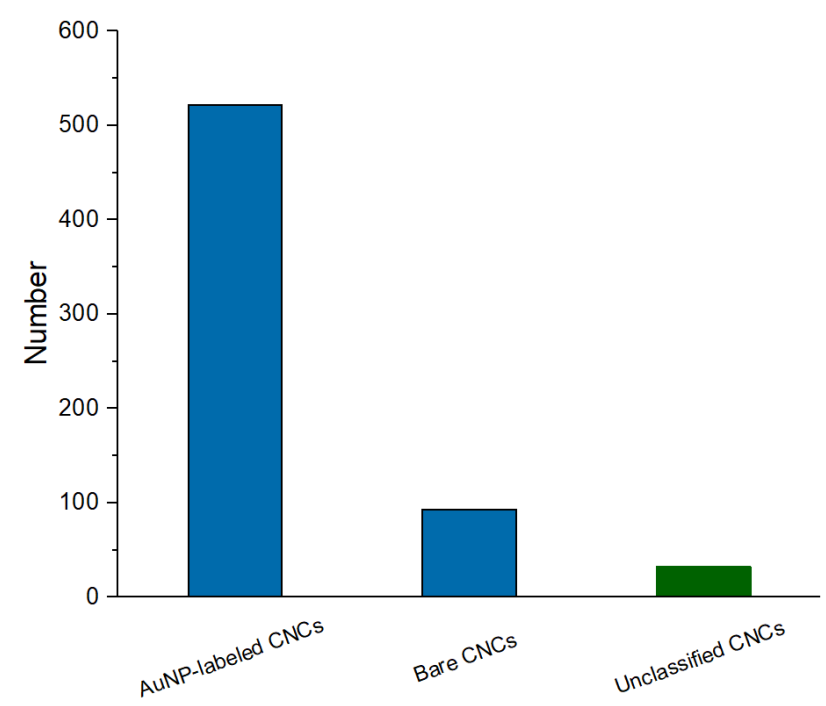

Figure 4. Number distribution of AuNP-labeled cCNCs and bare $C C N C s$ resulting from the in situ growth method in presence S-e-cCNCs. Particles that could not unambiguously be attributed to one or the other category appear as "unclassified". The histogram results from the analysis of about 650 particles.

Moreover, it is clear from the TEM images in Figures $3 c$ and $3 d$ that the AuNPs that were synthesized in the presence of S-e-cCNCs and grown on their end have a very low polydispersity. In order to get more information on that point, SAXS experiments were performed on a $1 \mathrm{wt}$. \% AuNP-ecCNCs suspension prepared by in situ growth. As shown in Figure 5, oscillations with scattering minima at $Q_{1}=0.055 \AA^{-1}$ and $Q_{2}=0.095 \AA^{-1}$, can be observed. Such features readily show the presence of welldefined objects. Since, as described in the Experimental Section, the contrast term between gold nanoparticles and solvent is much greater than between cellulose and solvent, the scattering intensity of AuNP-e-cCNCs particles in water is considered as arising from AuNPs only. Accordingly, assuming interaction-free particles due to the low concentration used, the experimental data were fitted using the form factor of dense spheres. As shown in Figure 5, a good quality of the fit was obtained, revealing the presence AuNP spheres of radius $7.6 \pm 1 \mathrm{~nm}$. This result thus confirms the presence of AuNPs with a low polydispersity, in full agreement with the TEM images. 


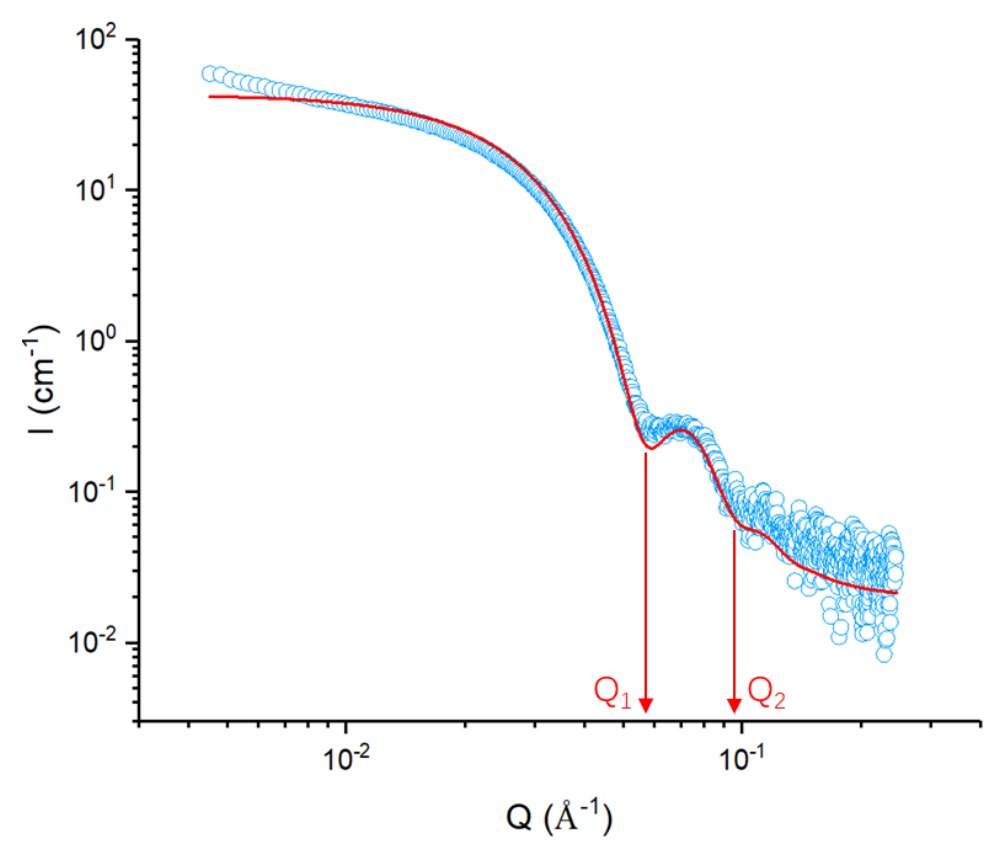

Figure 5. SAXS profile of a 1 wt. \% AuNP-e-cCNCs suspension obtained by the in situ growth method. The solid red straight line is a fit to the data using the form-factor of spheres of radius $7.6 \pm 1 \mathrm{~nm}$. See experimental section for details about the model.

UV-Vis spectroscopy experiments were performed on suspensions of AuNP-e-cCNCs synthesized by the two methods. The results shown in Supplementary Material Figure S5 first evidence that the plasmon resonance signal is the same for a pure suspension of AuNPs as for a suspension of AuNP-ecCNCs prepared by the direct reaction method, which demonstrates that this technique did not detect the formation of AuNP clusters. When the in situ growth strategy was used, the signal was shifted towards a lower wavelength (from 524 to $508 \mathrm{~nm}$ ), showing that the AuNPs that were synthesized in situ were smaller than the presynthesized ones, in agreement with DLS data and TEM images.

Overall, the different characterization techniques (DLS, TEM, SAXS and UV-Vis spectroscopy) concur to show the strong advantage in using the in situ growth approach that makes it possible to functionalize with a very high efficiency the reducing end of cCNCs with AuNPs exhibiting a low polydispersity, while avoiding the formation of AuNP clusters.

The in situ growth method was also applied to wCNCs. TEM images of AuNP-e-wCNCs distinctly show a spectacular asymmetric functionalization of single crystallite wCNCs on one end of the rods (Figure 6). A quite high attaching yield is observed even if the width, i.e. the reducing end surface of these wood CNCs is very low and inferior to that of cCNCs. The in situ growth method can therefore efficiently target the reducing end of CNCs even in the case where the end surface only represent a very small fraction of the whole rod surface. As in the case of cCNCs functionalization, a statistical 
analysis showed that about $70 \%$ of the wCNCs could be labeled with a gold nanoparticle (Supplementary Material Figure S6). The use of wCNCs constituted of only one or two elementary crystallites results in the majority of cases to the production of matchstick-like CNCs labeled with a single AuNP.

As shown in Supplementary Material Figure S7, DLS data for unlabeled and reducing end-labeled wCNCs are in agreement with TEM images. These results indeed confirm the presence after the in situ labeling reaction of a reduced amount of individual AuNPs, show that the hydrodynamic diameter of wCNC has increased from 140 to $164 \mathrm{~nm}$ after the labeling treatment and evidence the absence of aggregates. These data are in qualitative agreement with those obtained with cCNCs using the in situ growth method and confirm the high labeling efficiency achieved by this technique. UV-Vis data for AuNP-e-wCNCs in Supplementary Material Figure S5 show a plasmon resonance signal with a maximum at $515 \mathrm{~nm}$, in line with the presence of about $20 \mathrm{~nm}$ AuNPs.
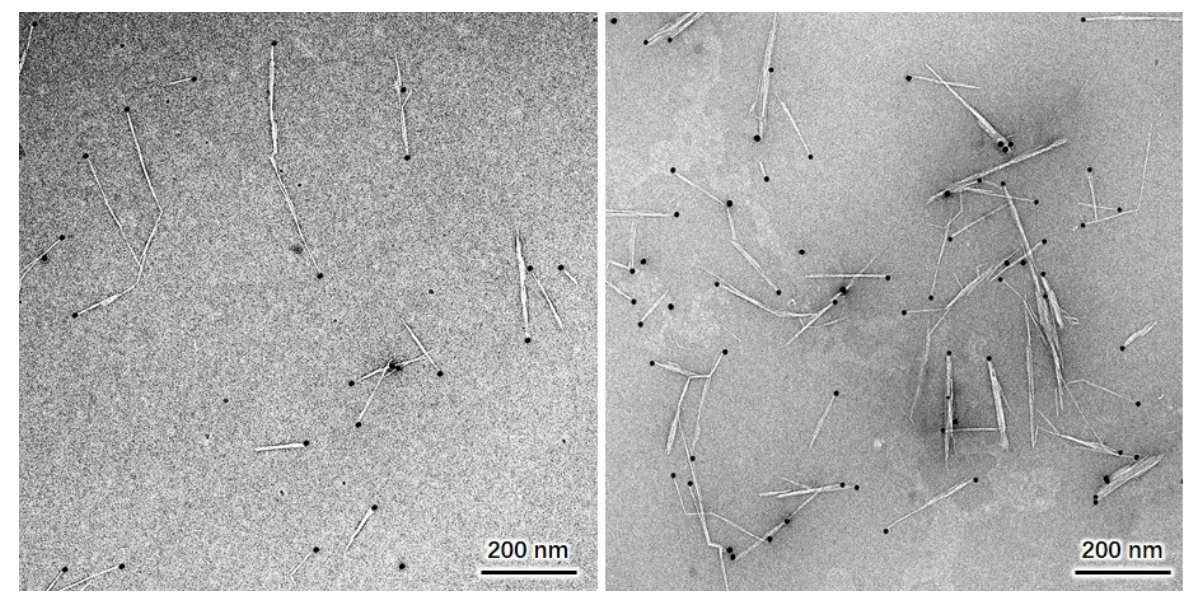

Figure 6. TEM images of negatively stained AuNP-e-wCNCs prepared by in situ growth from aqueous suspensions at room temperature.

\section{Discussion}

\subsection{Optimized labeling}

Even if the labeling of the CNC reducing end with gold or silver NPs has already been addressed by different groups based on the grafting of presynthesized particles, the labeling efficiency was significantly improved by using the in situ growth strategy described in the present work. While past works indeed reported maximum values of about $15 \%$ labelling efficiency, we achieved here up to $81 \%$ for CCNCs. In addition, the AuNPs grown in situ at the CNC reducing end exhibit a very narrow size distribution, as especially determined by SAXS. Importantly, the method was applied with a similar success to CNCs of two different origins, including wCNCs that possess a smaller cross-section than cCNCs, demonstrating a high degree of flexibility in the choice of the starting nanocrystals. Thus, the 
proposed method allowed us to overcome the synthesis challenge associated with the endwise modification of CNCs, which is related to their significantly low abundance, the small dimensions of the targeted reducing end sites and their limited reactivity. Data show that the nucleation and growth of AuNPs on sulfur-bearing reducing ends is an efficient way to increase the labeling efficiency while minimizing the AuNP polydispersity and formation of AuNP clusters. When compared to the grafting of presynthesized particles, the in situ growth technique does not suffer from limitations such as steric hindrance and electrostatic repulsions between similarly-charged CNCs and AuNPs but benefits from fast diffusion of the reactant in a water-soluble form. As a result, AuNPs preferentially nucleate and grow directly at the sulfur-derivatized reducing end, optimizing the labeling efficiency and limiting the synthesis of individual AuNPs and the presence of clusters. In absence of CNCs, the classical citrate method used to prepare AuNPs readily gives a fairly narrow size distribution centered around $20 \mathrm{~nm}$. AuNPs synthesized in presence of sulfur-functionalized CNCs exhibit a slightly more uniform size distribution that can tentatively arise from the sulfur-derivatized CNCs playing the role of stabilizing ligands since the presence of sulfur-containing ligands is known to promote the synthesis of AuNPs with a narrow size distribution (Daniel \& Astruc, 2004). Additionally, sulfur atoms at the CNC ends are preferential nucleation sites for gold clusters formation. Both effects contribute to lower the AuNP size distribution when compared with the synthesis in absence of pre-functionalized CNCs.

\subsection{Insights into the production and characteristics of cellulose nanocrystals}

Beyond this optimization that paves the way to the use of heavily functionalized CNCs for advanced applications, the data also contribute to give additional insight into more fundamental aspects, which are sometimes debated in the cellulose community.

Cellulose is synthesized at the plasma membrane by cellulose synthases that, in higher plants, are organized into rosette-like assemblies, acting as cellulose synthase spinnerets (Li et al., 2016; Pear, Kawagoe, Schreckengost, Delmer, \& Stalker, 1996). The cellulose chains in one cellulose microfibril are simultaneously spun by the rosette arrangement of cellulose synthases and due to the lack of solubility of the elongated chains, unidirectional cellulose crystallization occurs very shortly after the extrusion of the chains (Haigler, 1991). Due to the enzymatic synthesis, the nascent cellulose nanofibrils are oriented with the chains aligned in a parallel fashion (Li et al., 2016). Dried cellulose microfibrils used as a source for the production of CNCs contains alternating crystalline and more disordered regions. During hydrolysis, the acid preferentially etches away these less ordered regions, releasing shorter segments where the parallel packing of the chains is conserved, yielding rodlike particles with a chemical polarity. For CNCs made of a single elementary crystallite, all reducing ends are located on one end of the particle, as shown by crystallographic studies (Koyama, Helbert, et al., 1997; Nishiyama 
et al., 2002). This phenomenon was verified independently by using the selective cellulase Cel6A that degraded the non-reducing end of Valonia cellulose nanocrystals (Chanzy \& Henrissat, 1985) and by the gold nanoparticles labelling method on the reducing end of Valonia cellulose nanocrystals (Kim et al., 2006). In the present work, we could show that for commercial wCNCs originating from terrestrial plants and comprising one or two elementary crystallites, our in situ growth strategy successfully labeled only one end of the rods.

In contrast, the $\mathrm{CCNCS}$ were shown to be formed on average of 3 to 4 elementary subunits, which are laterally associated, forming flat objects having a width 3 to 4 times larger than their height (Elazzouzi-Hafraoui et al., 2008). This feature can easily be observed in Figure 1a and has been confirmed through the use of advanced techniques such as small-angle X-ray or neutron scattering (Azzam, Frka-Petesic, Semeraro, Cousin, \& Jean, 2020; Cherhal, Cousin, \& Capron, 2015). Such a composite nature of cCNCs has also been observed for $\mathrm{CNCs}$ extracted from other plants, e.g. bamboo (Brito et al., 2012). However, the respective orientation of these elementary units inside a CNC is not trivial and should depend on the way the CNCs were initially produced. A first possibility is that the acid hydrolysis isolates microfibril fragments composed of several neighboring crystallites without breaking the transverse hydrogen bonds connecting them, i.e. that the elementary crystallites constituting one CNC have never been separated during specimen preparation. In this case, the resulting nanocrystals have inherited the chemical polarity of their parent microfibrils and should be formed of chemically polar parallel crystallites, thus forming CNCs with all reducing ends of each crystallite located on the same side of the nanocrystal. In other words, with this scenario, the chemical polarity of the crystallites would be transferred to the $\mathrm{CNC}$ regrouping them.

A second hypothesis is that the acid hydrolysis is not only able to break the glycosidic linkages in the disordered regions along the nanofibrils, releasing shorter and more crystalline elements, but also to laterally separate the subunits, leading to individual single crystals that would subsequently reaggregate into the composite material observed. Upon re-aggregation, the respective orientation of the subunits within a CNC could be parallel, antiparallel, or random leading in the first case to reducing ends localized on the same side of the CNC and in the two other cases to reducing ends on both sides of the rod.

Figure 7 shows high-magnification TEM images of AuNP-labeled native cotton cellulose nanocrystals produced using the in situ growth method. As seen from these micrographs, AuNPs could successfully be bonded to one end of a majority of elementary subunits even though some elementary crystallites were not functionalized. Furthermore, when a CNC is considered, it is very clear that the AuNPs are all localized on the same side (there is no example of a cCNC tagged with AuNPs on both extremities). These results clearly show that elementary crystallites are oriented in a parallel manner 
in cCNCs with all reducing ends on the same side.

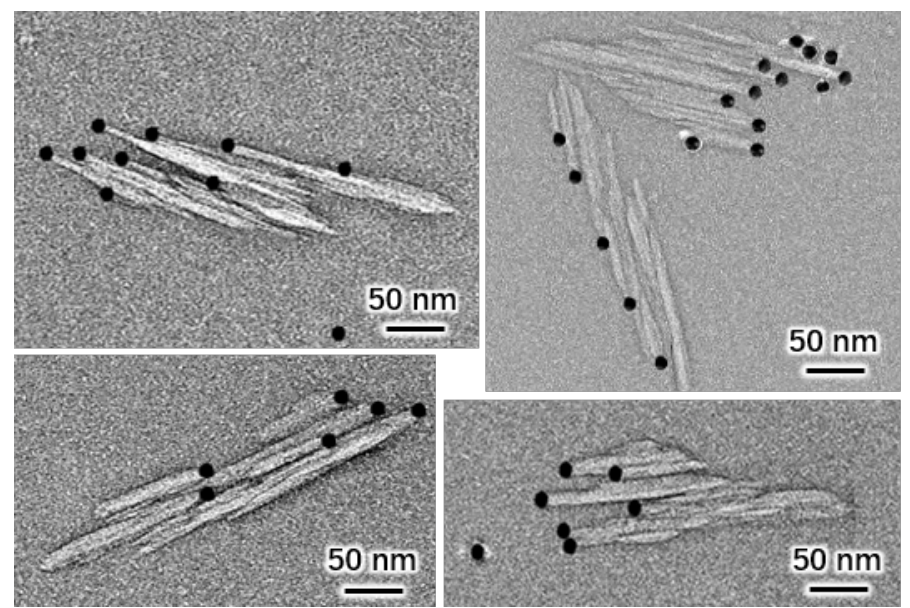

Figure 7. TEM micrographs of negatively stained AuNP-e-cCNCs prepared by in situ growth from aqueous suspensions at room temperature.

These data provide a clear visual proof that cCNCs formed by multiple laterally-associated crystallites are chemically polar. Flauzino Neto et al. concluded on a random antiparallel organization of elementary crystallites in native CNCs extracted from eucalyptus (Flauzino Neto et al., 2016). It seems that the case of cotton is different. It has to be noted that this conclusion is in full agreement with our previous study, where we reported the grafting of thermoresponsive polyetheramines at the reducing ends of $\mathrm{CCNCS}$ and the ability of these asymmetrically functionalized particles to assemble into star-shaped complexes (Lin et al., 2019). Indeed, since only star-like structures were formed and no end-to-end association was detected, the presence of reducing ends on both ends of the CNCs could be ruled out.

The localization of all reducing ends in composite cCNCs on a single side is consistent with our first hypothesis that the subunits in a CNC were never separated but does not strictly rule out the second hypothesis (re-aggregation of separated elementary crystallites) since even in this case a parallel reassembly is still possible, though not statistically probable. However, the fact that sulfuric acid hydrolysis not only individualizes the crystalline rods but also converts part of primary hydroxyl groups into sulfate half esters is in favor of the first speculation. Indeed, if individual elementary subunits would have been produced at some point during the hydrolysis, it would be expected that they have been imparted with these negative surface charges, which would have strongly hampered a possible re-association due to electrostatic repulsions. Consequently, we believe that the first CNC production scenario based on never-separated crystallites is more likely to take place than the second one involving separation and subsequent reaggregation of elementary crystallites.

Using TEM images of primary and secondary cotton cell walls or solid-state NMR in combination 
with spectral fitting, different groups distinctly showed that cotton microfibrils are present as tight bundles (Malm, Bulone, Wickholm, Larsson, \& Iversen, 2010; Willison \& Brown, 1977). The latter technique further revealed a width of the fibril aggregates of about $40 \mathrm{~nm}$, in fair agreement with the width of cCNCs. Based on these information and our results, it can be concluded that cCNCs directly arise from the isolation of the crystalline part of these fasciated parallely-arranged microfibril aggregates during the acid hydrolysis process. As it was also shown that the extent of fibril aggregation more generally depend on the cellulose source, drying and extraction processes (regulating the absence or presence of hemicelluloses and lignins)(Chunilall, Bush, Larsson, Iversen, \& Kindness, 2010; Hult, Iversen, \& Sugiyama, 2003), it can be assumed that wCNCs result from less associated fibrils.

\section{Conclusion}

Two different regioselective labeling strategies were applied to cellulose nanocrystals. The first method was a direct reaction in which CNCs prefunctionalized with sulfur atoms at the reducing end of the chains were mixed with pre-synthesized gold nanoparticles. As shown by DLS and TEM, such a strategy results in a low labeling ratio and the presence of gold nanoparticles aggregates. A second method, so-called in situ growth functionalization strategy, was applied, where AuNPs were synthesized from soluble derivatives in the presence of sulfur-bearing CNCs. In this case, a very high labeling efficiency of about $80 \%$ was reached for CNCs prepared from wood or cotton. A statisticallyrelevant analysis of the TEM images further evidenced that one or several AuNPs were located on one end of the rods and never on two opposite ends. Furthermore, there were nearly no gold nanoparticles aggregates and these in situ-synthesized AuNPs had a very low polydispersity. All complementary techniques used, namely TEM, DLS, UV-Vis spectroscopy and SAXS spectra, supported these findings. This optimized labeling strategy was subsequently used to address questions about the constitution of CNCs. Our results evidenced that in native cotton CNCs made of the lateral assembly of a few elementary crystallites, these crystallites are arranged in a parallel (unidirectional) manner with all reducing ends located on the same side of the nanocrystals. This organization showed that the acid hydrolysis used to prepare the cotton nanocrystals isolated fragments from preexisting assemblies of parallelly-packed microfibrils, without laterally separating them.

\section{Acknowledgements.}

This work was developed in the framework of Glyco@Alps (ANR-15-IDEX-02). The authors acknowledge the NanoBio chemistry platform (ICMG FR 2607) for granting access to the electron microscopy facilities and thank Fabrice Cousin for SAXS measurements performed at the SWAXS lab (LLB, CEA Saclay). The authors are grateful to Henri Chanzy for constant support, fruitful discussions and 


\section{References}

Angles, M. N., \& Dufresne, A. (2001). Plasticized starch/tunicin whiskers nanocomposite materials. 2. Mechanical behavior. Macromolecules, 34(9), 2921-2931.

Azzam, F., Frka-Petesic, B., Semeraro, E. F., Cousin, F., \& Jean, B. (2020). Small-Angle Neutron Scattering Reveals the Structural Details of Thermosensitive Polymer-Grafted Cellulose Nanocrystal Suspensions. Langmuir, 36(29), 8511-8519.

Bondeson, D., Mathew, A., \& Oksman, K. (2006). Optimization of the isolation of nanocrystals from microcrystalline cellulose by acid hydrolysis. Cellulose, 13(2), 171-180.

Bras, J., \& Chauve, G. (2014). Industrial point of view of nanocellulose materials and their possible applications. In handbook of green materials: Processing technologies, properties and applications. World Sci. Pub. Co.

Brito, B. S. L., Pereira, F. V., Putaux, J. L., \& Jean, B. (2012). Preparation, morphology and structure of cellulose nanocrystals from bamboo fibers. Cellulose, 19(5), 1527-1536.

Chanzy, H., \& Henrissat, B. (1985). Undirectional degradation of valonia cellulose microcrystals subjected to cellulase action. FEBS Letters, 184(2), 285-288.

Chen, L., Cao, W. J., Quinlan, P. J., Berry, R. M., \& Tam, K. C. (2015). Sustainable catalysts from gold-loaded polyamidoamine dendrimer-cellulose nanocrystals. ACS Sustainable Chemistry \& Engineering, 3(5), 978-985.

Cherhal, F., Cousin, F., \& Capron, I. (2015). Influence of Charge Density and lonic Strength on the Aggregation Process of Cellulose Nanocrystals in Aqueous Suspension, as Revealed by SmallAngle Neutron Scattering. Langmuir, 31(20), 5596-5602.

Chunilall, V., Bush, T., Larsson, P. T., Iversen, T., \& Kindness, A. (2010). A CP/MAS 13C-NMR study of cellulose fibril aggregation in eucalyptus dissolving pulps during drying and the correlation between aggregate dimensions and chemical reactivity. Holzforschung, 64(6), 693-698.

Daniel, M. C. \& Astruc, D. (2004). Gold Nanoparticles: Assembly, Supramolecular Chemistry, Quantum-SizeRelated Properties, and Applications toward Biology, Catalysis, and Nanotechnology. Chem. Rev., 104, 293-346.

Dufresne, A. (2012). Nanocellulose: from nature to high performance tailored materials. Berlin: Walter de Gruyter $\mathrm{GmbH}$.

Eichhorn, S. J., Dufresne, A., Aranguren, M., Marcovich, N. E., Capadona, J. R., Rowan, S. J., Weder, C., Thielemans, W., Roman, M., Renneckar, S., Gindl, W., Veigel, S., Keckes, J., Yano, H., Abe, K., Nogi, M., Nakagaito, A. N., Mangalam, A., Simonsen, J., Benight, A. S., Bismarck, A., Berglund, L. A., \& Peijs, T. (2010). Review: current international research into cellulose nanofibres and nanocomposites. Journal of Materials Science, 45(1), 1-33.

Eichhorn, S. J., Young, R. J., \& Davies, G. R. (2005). Modeling crystal and molecular deformation in regenerated cellulose fibers. Biomacromolecules, 6(1), 507-513.

Elazzouzi-Hafraoui, S., Nishiyama, Y., Putaux, J.-L., Heux, L., Dubreuil, F., \& Rochas, C. (2008). The shape and size distribution of crystalline nanoparticles prepared by acid hydrolysis of native cellulose. Biomacromolecules, 9(1), 57-65.

Flauzino Neto, W. P., Putaux, J.-L., Mariano, M., Ogawa, Y., Otaguro, H., Pasquini, D., \& Dufresne, A. (2016). Comprehensive morphological and structural investigation of cellulose I and II nanocrystals prepared by sulphuric acid hydrolysis. RSC Advances, 6(79), 76017-76027.

Habibi, Y., Lucia, L. A., \& Rojas, O. J. (2010). Cellulose nanocrystals: chemistry, self-assembly, and applications. Chemical Reviews, 110(6), 3479-3500.

Haigler, C. H. (1991). Biosynthesis and biodegradation of cellulose. New York: Marcel Dekker.

Haiss, W., Thanh, N. T. K., Aveyard, J., \& Fernig, D. G. (2007). Determination of size and concentration of 
gold nanoparticles from UV-Vis spectra. Analytical Chemistry, 79(11), 4215-4221.

Heise, K., Delepierre, G., King, A. W. T., Kostiainen, M. A., Zoppe, J. O., Weder, C., \& Kontturi, E. (2020). Chemical modification of cellulose nanocrystal reducing end groups. Angewandte ChemieInternational Edition.

Hieta, K., Kuga, S., \& Usuda, M. (1984). Electron stainning of reducing ends evidences a parallel-chain structure in valonia cellulose. Biopolymers, 23(10), 1807-1810.

Hult, E.-L., Iversen, T., \& Sugiyama, J. (2003). Characterization of the supermolecular structure of cellulose in wood pulp fibres. Cellulose, 10(2), 103-110.

Kim, N. H., Imai, T., Wada, M., \& Sugiyama, J. (2006). Molecular directionality in cellulose polymorphs. Biomacromolecules, 7(1), 274-280.

Klemm, D., Cranston, E. D., Fischer, D., Gama, M., Kedzior, S. A., Kralisch, D., Kramer, F., Kondo, T., Lindstrom, T., Nietzsche, S., Petzold-Welcke, K., \& Rauchfuss, F. (2018). Nanocellulose as a natural source for groundbreaking applications in materials science: Today's state. Materials Today, 21(7), 720-748.

Koyama, M., Helbert, W., Imai, T., Sugiyama, J., \& Henrissat, B. (1997). Parallel-up structure evidences the molecular directionality during biosynthesis of bacterial cellulose. Proceedings of the National Academy of Sciences of the United States of America, 94(17), 9091-9095.

Koyama, M., Sugiyama, J., \& Itoh, T. (1997). Systematic survey on crystalline features of algal celluloses. Cellulose, 4(2), 147-160.

Kuga, S., \& Brown, R. M. (1988). Silver labeling of the reducing ends of bacterial cellulose. Carbohydrate Research, 180(2), 345-350.

Lagerwall, J. P. F., Schutz, C., Salajkova, M., Noh, J., Park, J. H., Scalia, G., \& Bergstrom, L. (2014). Cellulose nanocrystal-based materials: from liquid crystal self-assembly and glass formation to multifunctional thin films. NPG Asia Materials, 6, 12.

Li, L., Tao, H., Wu, B. L., Zhu, G., Li, K., \& Lin, N. (2018). Triazole end-grafting on cellulose nanocrystals for water-redispersion improvement and reactive enhancement to nanocomposites. ACS Sustainable Chemistry \& Engineering, 6(11), 14888-14900.

Li, S. D., Bashline, L., Zheng, Y. Z., Xin, X. R., Huang, S. X., Kong, Z. S., Kim, S. H., Cosgrove, D. J., \& Gu, Y. (2016). Cellulose synthase complexes act in a concerted fashion to synthesize highly aggregated cellulose in secondary cell walls of plants. Proceedings of the National Academy of Sciences of the United States of America, 113(40), 11348-11353.

Lin, F. B., Cousin, F., Putaux, J.-L., \& Jean, B. (2019). Temperature-controlled star-shaped cellulose nanocrystal assemblies resulting from asymmetric polymer grafting. ACS Macro Letters, 8(4), 345351.

Lokanathan, A. R., Lundahl, M., Rojas, O. J., \& Laine, J. (2014). Asymmetric cellulose nanocrystals: thiolation of reducing end groups via NHS-EDC coupling. Cellulose, 21(6), 4209-4218.

Lokanathan, A. R., Nykanen, A., Seitsonen, J., Johansson, L. S., Campbell, J., Rojas, O. J., Ikkala, O., \& Laine, J. (2013). Cilia-mimetic hairy surfaces based on end-immobilized nanocellulose colloidal rods. Biomacromolecules, 14(8), 2807-2813.

Malm, E., Bulone, V., Wickholm, K., Larsson, P. T., \& Iversen, T. (2010). The surface structure of well-ordered native cellulose fibrils in contact with water. Carbohydrate Research, 345(1), 97-100.

Ning, Y. G., Xie, H., Xing, H. T., Deng, W. L., \& Yang, D. B. (1996). Comparison of self-assembled monolayers of $\mathrm{n}$-alkanethiols and phenylthioureas on the surface of gold. Surface and Interface Analysis, 24(9), 667-670.

Nishiyama, Y., Langan, P., \& Chanzy, H. (2002). Crystal structure and hydrogen-bonding system in cellulose 1 beta from synchrotron $\mathrm{X}$-ray and neutron fiber diffraction. Journal of the American Chemical Society, 124(31), 9074-9082.

Pear, J. R., Kawagoe, Y., Schreckengost, W. E., Delmer, D. P., \& Stalker, D. M. (1996). Higher plants contain homologs of the bacterial celA genes encoding the catalytic subunit of cellulose synthase. Proceedings of the National Academy of Sciences of the United States of America, 93(22), 1263712642.

Revol, J. F., Bradford, H., Giasson, J., Marchessault, R. H., \& Gray, D. G. (1992). Helicoidal self-ordering of 
cellulose microfibrils in aqueous suspension. International Journal of Biological Macromolecules, 14(3), 170-172.

Rivkin, A., Abitbol, T., Nevo, Y., Verker, R., Lapidot, S., Komarov, A., Veldhuis, S. C., Ziberman, G., Reches, M., Cranston, E. D., \& Shoseyov, O. (2015). Bionanocomposite Films from Resilin-CBD Bound to Cellulose Nanocrystals. Industrial Biotechnology, 11(1), 44-58.

Ruiz, M. M., Cavaille, J. Y., Dufresne, A., Gerard, J. F., \& Graillat, C. (2000). Processing and characterization of new thermoset nanocomposites based on cellulose whiskers. Composite Interfaces, 7(2), 117131.

Semenikhin, N. S., Kadasala, N. R., Moon, R. J., Perry, J. W., \& Sandhage, K. H. (2018). Individually dispersed gold nanoshell-bearing cellulose nanocrystals with tailorable plasmon resonance. Langmuir, 34(15), 4427-4436.

Shi, Z. Q., Tang, J. T., Chen, L., Yan, C. R., Tanvir, S., Anderson, W. A., Berry, R. M., \& Tam, K. C. (2015). Enhanced colloidal stability and antibacterial performance of silver nanoparticles/cellulose nanocrystal hybrids. Journal of Materials Chemistry B, 3(4), 603-611.

Sturcova, A., Davies, G. R., \& Eichhorn, S. J. (2005). Elastic modulus and stress-transfer properties of tunicate cellulose whiskers. Biomacromolecules, 6(2), 1055-1061.

Tang, J. T., Shi, Z. Q., Berry, R. M., \& Tam, K. C. (2015). Mussel-inspired green metallization of silver nanoparticles on cellulose nanocrystals and their enhanced catalytic reduction of 4-nitrophenol in the presence of beta-cyclodextrin. Industrial \& Engineering Chemistry Research, 54(13), 3299-3308.

Tao, H., Lavoine, N., Jiang, F., Tang, J. T., \& Lin, N. (2020). Reducing end modification on cellulose nanocrystals: strategy, characterization, applications and challenges. Nanoscale Horizons, 5(4), 607-627.

Ulman, A. (1996). Formation and structure of self-assembled monolayers. Chemical Reviews, 96(4), 15331554.

Vollhardt, K. P. C., \& Schore, N. E. (2014). Organic chemistry. Macmillan International Higher Education.

Willison, J., \& Brown, R. M. (1977). An examination of the developing cotton fiber: wall and plasmalemma. Protoplasma, 92(1-2), 21-41.

Wu, X. D., Lu, C. H., Zhang, W., Yuan, G. P., Xiong, R., \& Zhang, X. X. (2013). A novel reagentless approach for synthesizing cellulose nanocrystal-supported palladium nanoparticles with enhanced catalytic performance. Journal of Materials Chemistry A, 1(30), 8645-8652.

Wu, X. D., Lu, C. H., Zhou, Z. H., Yuan, G. P., Xiong, R., \& Zhang, X. X. (2014). Green synthesis and formation mechanism of cellulose nanocrystal-supported gold nanoparticles with enhanced catalytic performance. Environmental Science-Nano, 1(1), 71-79.

Yang, X., Shi, K. Y., Zhitomirsky, I., \& Cranston, E. D. (2015). Cellulose nanocrystal aerogels as universal 3D lightweight substrates for supercapacitor materials. Advanced Materials, 27(40), 6104-6109.

Yokota, S., Kitaoka, T., Opietnik, M., Rosenau, T., \& Wariishi, H. (2008). Synthesis of gold nanoparticles for in situ conjugation with structural carbohydrates. Angewandte Chemie-International Edition, 47(51), 9866-9869.

Zhang, Z., Sebe, G., Wang, X. S., \& Tam, K. C. (2018). Gold nanoparticles stabilized by poly(4-vinylpyridine) grafted cellulose nanocrystals as efficient and recyclable catalysts. Carbohydrate Polymers, 182, 61-68. 